\title{
Genomic and transcriptomic analyses reveal polygenic architecture for ecologically-important functional traits in aspen (Populus tremuloides Michx.)
}

\author{
Jennifer Lind-Riehl ${ }^{1}$, Christopher Cole ${ }^{2}$, Clay Morrow ${ }^{1}$, Hilary Barker ${ }^{1}$, Carolina \\ Bernhardsson $^{3}$, Kennedy Rubert-Nason ${ }^{2}$, Pär Ingvarsson ${ }^{4}$, and Richard Lindroth ${ }^{5}$ \\ ${ }^{1}$ University of Wisconsin-Madison \\ ${ }^{2}$ University of Wisconsin Madison \\ ${ }^{3}$ Uppsala Universitet \\ ${ }^{4}$ Sveriges lantbruksuniversitet \\ ${ }^{5}$ University of Wisconsin Madison Graduate School
}

February 10, 2022

\begin{abstract}
Intraspecific genetic variation in foundation species such as trembling aspen shapes their impact on forest structure and function. Identifying genes and genomic regions underlying ecologically relevant traits is key to understanding that impact. Previous studies using genome-wide association (GWA) analyses to identify candidate genes have identified fewer genes than anticipated for highly heritable traits. Mounting evidence suggests that polygenic control of quantitative traits is largely responsible for this "missing heritability" phenomenon. Our research characterized the genetic architecture of 40 functional traits using genomic and transcriptomic analyses in an association mapping population of aspen. A multi-marker association model revealed that most traits displayed a polygenic architecture, with most variation explained by loci with small effects (below the detection levels of single-marker GWA methods). Consistent with a polygenic architecture, our single-marker GWA analyses found only 35 significant SNPs in 22 genes across 15 trait/trait combinations. Next, we used differential expression analysis on a subset of aspen genets with divergent concentrations of salicinoid phenolic glycosides (key defense traits). This alternative method to traditional GWA discovered 1,243 differentially expressed genes for a polygenic trait. Soft clustering analysis revealed three gene clusters (246 candidate genes) involved in secondary metabolite biosynthesis and regulation. Our results support the omnigenic model that complex traits are largely controlled by many small effect loci, most of which may not have obvious connections to the traits of interest. Our work reveals that functional traits governing higher-order community- and ecosystem-level attributes of a foundation forest tree species have complex underlying genetic structures and will require methods beyond traditional GWA analyses to unravel.
\end{abstract}

\section{Hosted file}

MEC_Manuscript_Final.doc available at https://authorea.com/users/459862/articles/555983genomic-and-transcriptomic-analyses-reveal-polygenic-architecture-for-ecologically-

important-functional-traits-in-aspen-populus-tremuloides-michx 

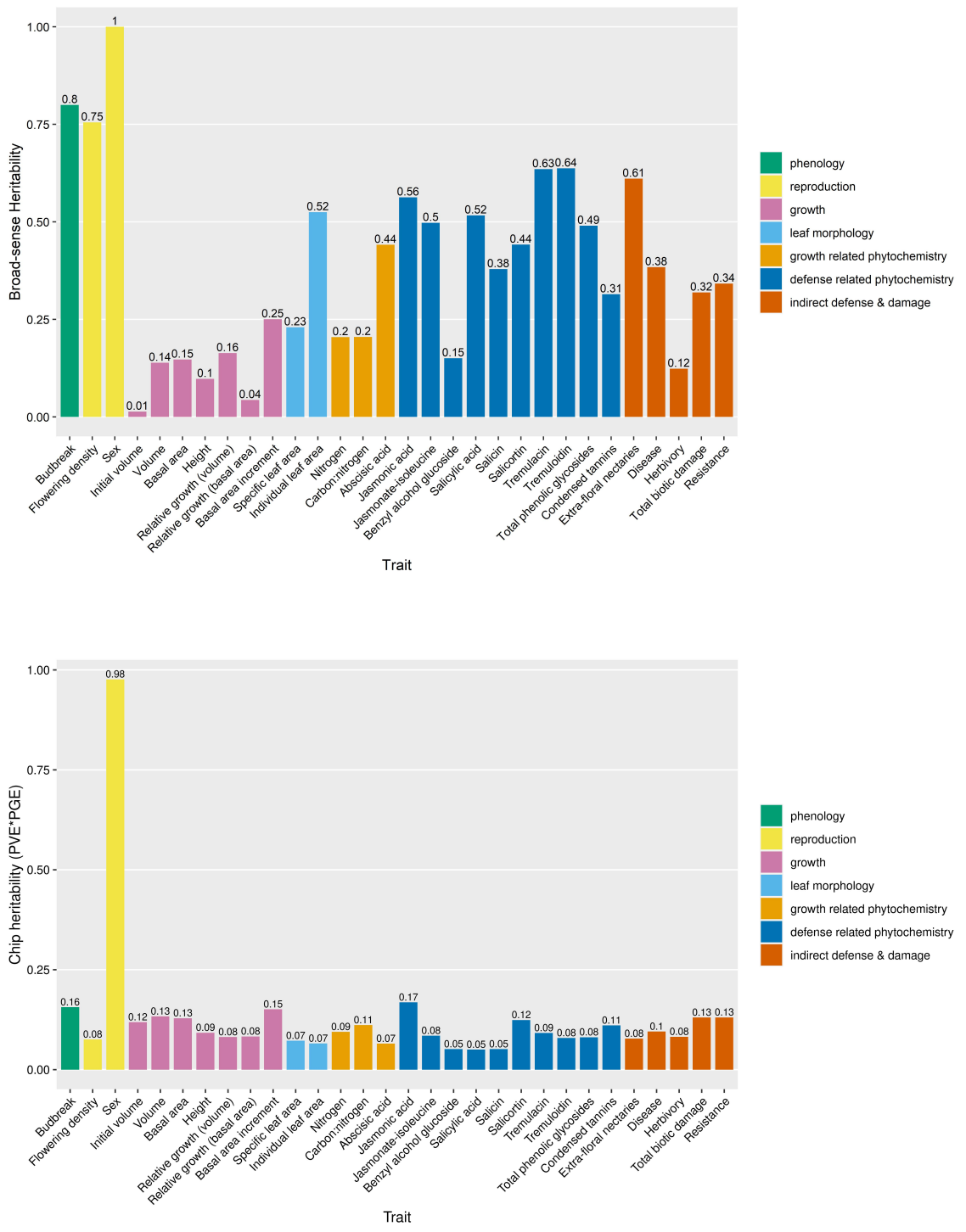

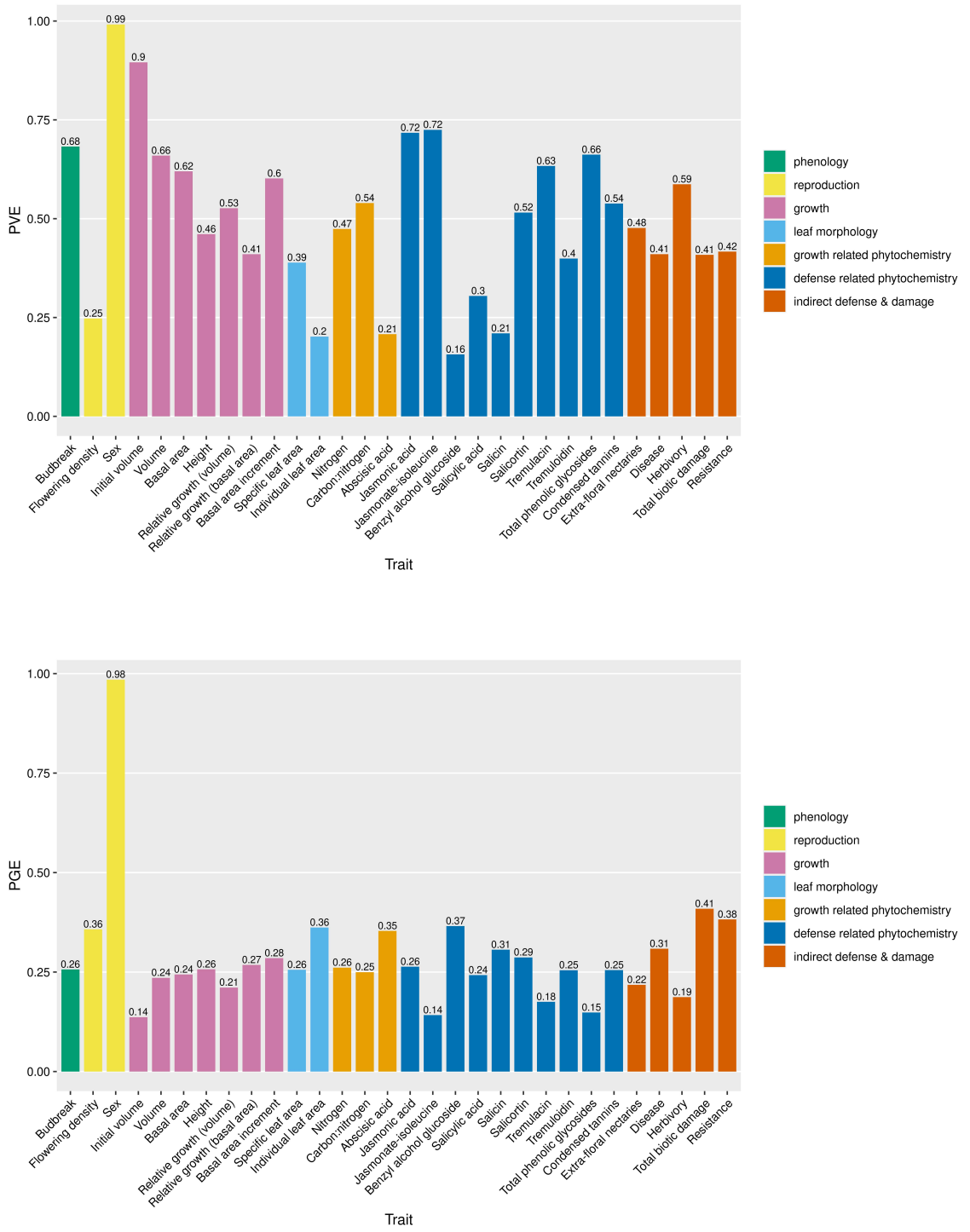
- Log2 FC - adjusted p-value \& Log2 FC
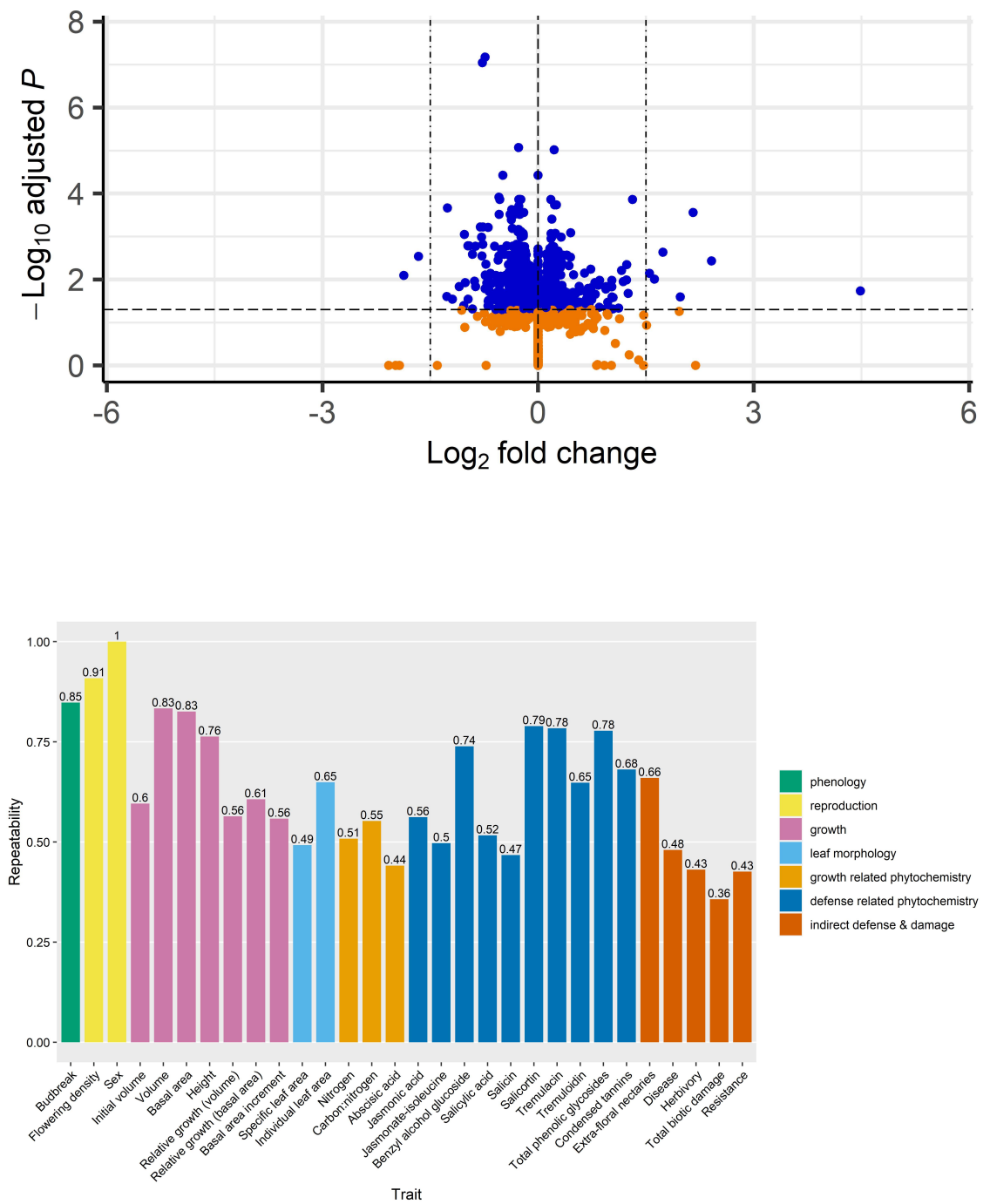

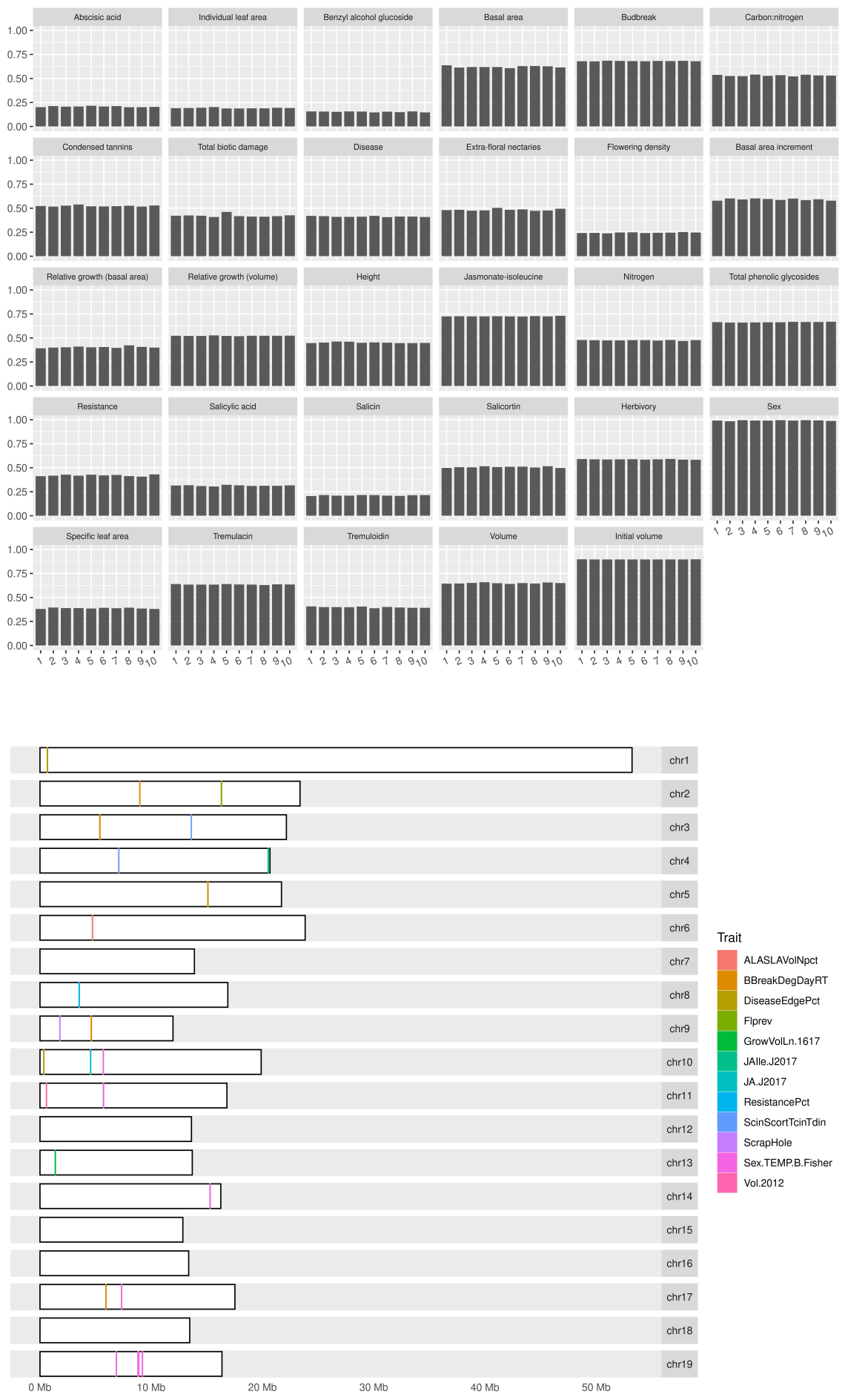


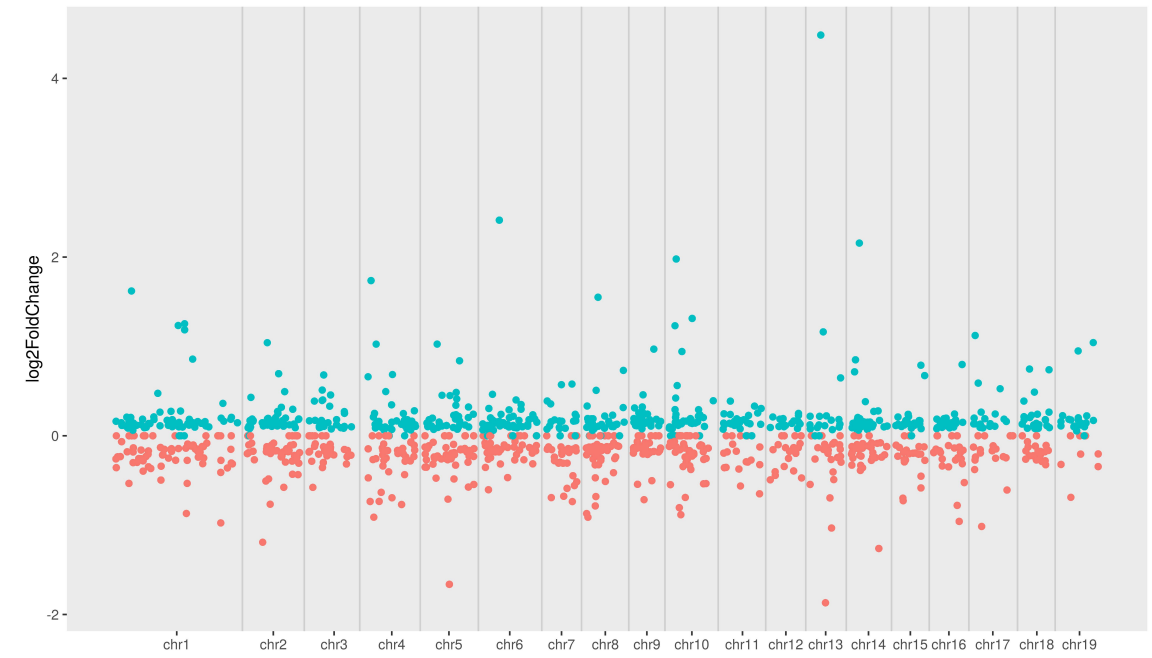

